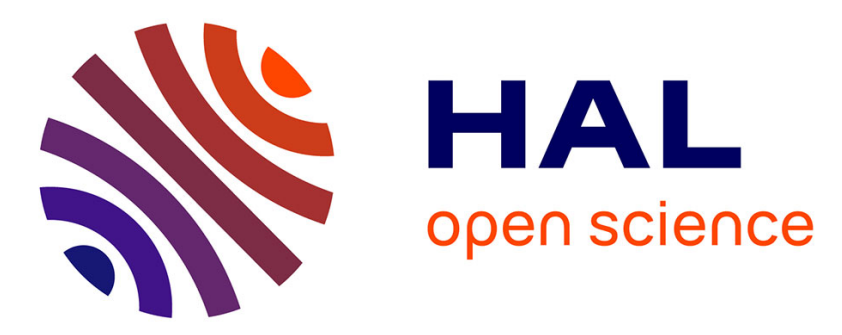

\title{
In Vivo Achilles Tendon Elasticity Assessment using Supersonic Shear Imaging: a feasibility study
}

\author{
J Brum, M Bernal, M Fink, Jean Luc Gennisson, M Tanter
}

\section{To cite this version:}

J Brum, M Bernal, M Fink, Jean Luc Gennisson, M Tanter. In Vivo Achilles Tendon Elasticity Assessment using Supersonic Shear Imaging: a feasibility study. 2013 IEEE International Ultrasonics Symposium (IUS), Jul 2013, Prague, Czech Republic. 10.1109/ULTSYM.2013.0299 hal-03275147

\section{HAL Id: hal-03275147 \\ https://hal.science/hal-03275147}

Submitted on 30 Jun 2021

HAL is a multi-disciplinary open access archive for the deposit and dissemination of scientific research documents, whether they are published or not. The documents may come from teaching and research institutions in France or abroad, or from public or private research centers.
L'archive ouverte pluridisciplinaire HAL, est destinée au dépôt et à la diffusion de documents scientifiques de niveau recherche, publiés ou non, émanant des établissements d'enseignement et de recherche français ou étrangers, des laboratoires publics ou privés. 


\title{
In Vivo Achilles Tendon Elasticity Assessment using Supersonic Shear Imaging: a feasibility study
}

\author{
J. Brum, M. Bernal, M. Fink, J.L. Gennisson, M. Tanter \\ Institut Langevin - Ondes et Images \\ ESPCI ParisTech, CNRS UMR 7587, Inserm ERL U979 \\ Paris, France \\ javier.brum@espci.fr
}

\begin{abstract}
In this study shear wave dispersion was measured in vivo in four Achilles tendons by applying the Supersonic Shear Imaging technique and the concept of Shear Wave Spectroscopy parallel and perpendicular to the tendon fiber orientation. In tissues shear wave dispersion is related to two main physical causes: the geometry of the investigated medium which can lead to guided waves and/or the viscosity of the medium (if Voigt model is assumed). By modeling the Achilles tendon as a transverse isotropic plate it was possible to estimate the tendon elastic properties described by the five independent elastic constants $\left(\mathbf{C}_{11}, \mathbf{C}_{33}, \mathbf{C}_{13}, \mathbf{C}_{55}\right.$ and $\left.\mathbf{C}_{66}\right)$ of the Christoffel's tensor. We show that parallel to fibers the geometrical effect leading to guided wave propagation is prominent, while perpendicularly to fibers viscosity must be also taken into account to fully describe the experimental data. The obtained results agree with those reported in the literature. In this study we have demonstrated that the use of a dispersion model must be taken into account in order to properly estimate the tendon viscoelastic properties.
\end{abstract}

Keywords-Achilles tendon, transverse isotropy, Lamb waves, viscoelasticity, supersonic shear imaging, shear wave spectroscopy

\section{INTRODUCTION}

Common tendon disorders (e.g. Achilles tendon rupture or flexor tendon injury) are responsible for substantial morbidity in sports and daily life. Repetitive tendon overload result in a degenerative condition called tendinopathy and its treatment seeks to restore the tendon mechanical functions. Thus, noninvasive evaluation of the tendon's elastic properties is of great importance to predict tendon rupture and in the follow up during recovery treatments. However, unsatisfactory results are common and the mechanical properties of the injured tendon are hard to assess quantitatively during the recovery process. As a consequence, a non-invasive tool capable of quantifying tendon stiffness is of great need clinically.

Several authors have characterized tendon stiffness and structure [1]-[3]. Usually, tendons may be described as a unidirectional arrangement of collagen fibers within a supporting matrix. Thus, the isotropic solid model is no longer valid and a transverse isotropic model is more suitable. Kuo et al. [2] evaluated anisotropy in vitro on six fresh bovine Achilles tendons using ultrasound speed measurements along different propagation directions. In [2] Young's modulus of $134 \mathrm{kPa}$ and $35 \mathrm{kPa}$ were obtained parallel and perpendicular to the fiber orientation respectively. Recently, Aubry et al. [3] evidenced anisotropy by applying the Supersonic Shear Imaging (SSI) technique in vivo to human Achilles tendons. By estimating the shear wave time of flight, shear wave velocities of $\sim 15 \mathrm{~m} / \mathrm{s}$ and $\sim 5 \mathrm{~m} / \mathrm{s}$ were measured parallel and perpendicular to the fiber orientation respectively. Given these values and the frequency range used for the SSI technique (300 $-800 \mathrm{~Hz}$ ), the shear wave wavelengths are greater than the mean tendon thickness $(\sim 4 \mathrm{~mm})$ [3]. Thus, shear waves may be guided along and across the tendon as Lamb waves as it has already been observed in cornea [4] and arteries [5]. Therefore, to avoid underestimation on the shear wave speed calculated from the shear wave time of flight, a dispersion analysis is required.

The aim of this work is to adapt the SSI technique to map in vivo the mechanical properties of extremely stiff and geometrically complex media as tendons. To this end, shear wave propagation in a direction parallel and perpendicular to the fiber orientation is studied as a function of frequency by using Shear Wave Spectroscopy (SWS) [6].

\section{MATERIALS AND METHODS}

\section{A. Experimental Procedure}

The left and right tendons of two healthy volunteers were studied. For each experiment the volunteer was in a sitting position with a $90^{\circ}$ angle between the foot and the tibia as well as between the tibia and the femur as presented in Fig. 1. The foot and the ultrasonic probe were submerged in a water tank to avoid pre-compression of the tendon and to facilitate the positioning of the probe. Once a proper B-Mode image of the tendon was achieved the probe position was held fixed through an articulated arm. Then, the SSI technique was applied to the tendon either parallel or perpendicular to the fibers by tilting the probe by a $90^{\circ}$ angle.

\section{B. Supersonic Shear Imaging (SSI) Technique}

For the SSI technique [7], the ultrasonic radiation force is used as shear wave source. An ultrafast ultrasound scanner (Aixplorer, Supersonic Imagine, Aix en Provence, France) is used to drive a conventional 256 elements, $8 \mathrm{MHz}$ ultrasonic linear array (SL15-4, Supersonic Imagine). A pushing sequence consisting of three to four pushing points $(150 \mu \mathrm{s}$ 
duration each) at different depths, centered on the tendon position, is used to generate a broadband low frequency shear wave $(300-800 \mathrm{~Hz})$ which propagates in a direction perpendicular to the ultrasonic beam. The system is then switched to an ultrafast ultrasound imaging mode to image the shear wave propagation at a $15 \mathrm{kHz}$ rate.

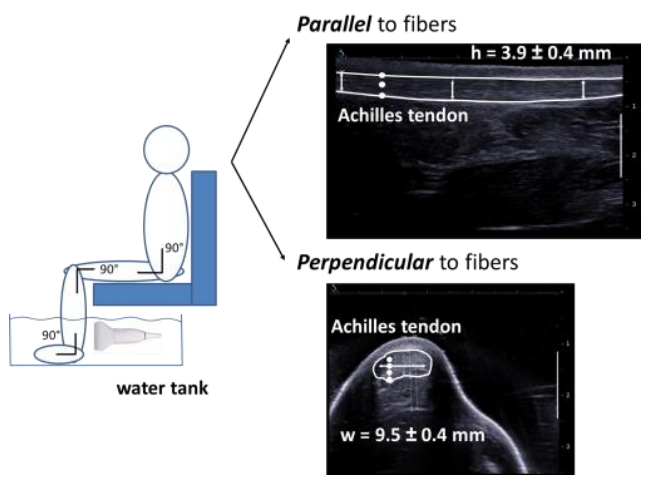

Fig. 1. Representation of the experimental procedure used for the Achilles tendon elasticity assesment. B-Mode images of the tendon (indicated with white lines) for the probe position parallel and perpendicular to the fibers are also presented (pushing points are indicated in white dots).

\section{EXPERIMENTAL RESULTS AND INTERPRETATION}

\section{A. Shear Wave Spectroscopy (SWS) in the Achilles tendon}

From the measured displacement movie it is possible to extract the shear wave dispersion curve (i.e. relation between phase velocity and frequency). The first step consists in transforming the $(\mathrm{x}, \mathrm{z}, \mathrm{t})$ displacement movie into a two dimensional data set $(\mathrm{x}, \mathrm{t})$ by looking at a fixed depth inside the tendon. Then, by applying a 2-D Fourier transform to the $(x, t)$ displacement it is possible to extract the wave dispersion curve by finding at each frequency, the phase velocity at which the Fourier transform amplitude is maximal. The procedure is represented in Fig. 2. For the final dispersion curve an average over the tendon thickness is performed. Results for each subject are presented in Fig. 3 and Fig. 4 for the parallel and perpendicular direction respectively. The error bar is provided by the standard deviation of the dispersion curve over the tendon thickness.

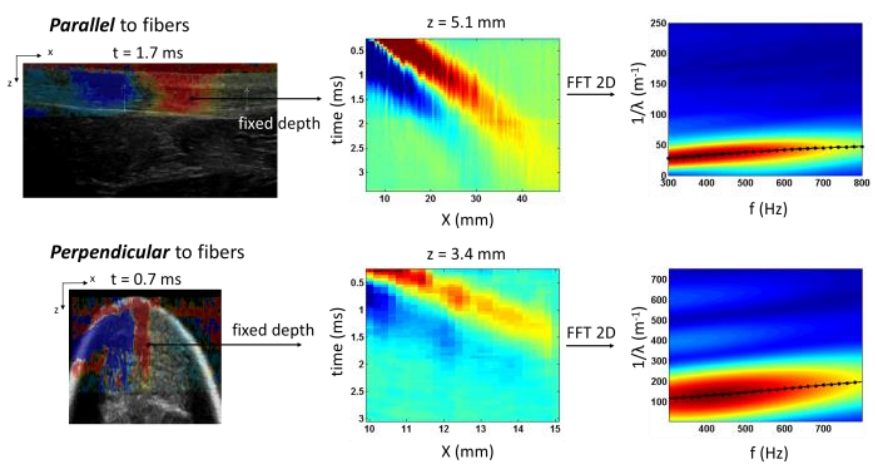

Fig. 2. Shear wave spectroscopy in the Achilles tendon for the shear wave propagation direction parallel and perpendicular to the fibers.
Similar behavior is observed for all four tendons. Phase velocity values between $12.9 \pm 1.7 \mathrm{~m} / \mathrm{s}$ and $16.9 \pm 2.1 \mathrm{~m} / \mathrm{s}$ were measured for frequencies between 300 and $800 \mathrm{~Hz}$ parallel to the fiber orientation (mean value \pm standard deviation over the four tendons). In the perpendicular direction the values ranged from $3.1 \pm 0.5 \mathrm{~m} / \mathrm{s}$ to $4.3 \pm 0.6 \mathrm{~m} / \mathrm{s}$ for the same bandwidth mentioned above. It is important to note that the values obtained in our study coincide with those reported in the literature [3]. However, non-negligible dispersive effects are observed for wave propagation in both directions. Thus, a dispersion model should be taken into account in order to estimate the tendon's elastic properties.

Shear wave dispersion has already been observed in tissues due to viscosity (e.g. muscle or liver) [6], [8], guided wave propagation (e.g. cornea or arteries) [4], [5] or both (e.g. myocardium) [9]. In the case of the Achilles tendon, based on the measured phase velocity at $500 \mathrm{~Hz}$, wavelengths of $25 \mathrm{~mm}$ and $7 \mathrm{~mm}$ are calculated for the parallel and perpendicular direction respectively, which are greater than the mean tendon thickness ( $h \sim 4 \mathrm{~mm}$ in Fig. 1). Thus, in a first approximation, guided wave propagation is assumed to be responsible for shear wave dispersion in the Achilles tendon. Furthermore, due to the ratio between parallel and perpendicular phase velocities $(\sim 3)$ and the ratio between tendon width and thickness $(w / h$ $\sim 3)$, wave propagation along parallel and perpendicular directions does not interfere with each other. As a consequence, wave propagation along both directions is assumed to take place in a transverse isotropic plate. The plate is assumed to be surrounded by liquid (leaky Lamb waves) as it was done in [4], [5] and [9] for other tissues.

\section{B. Achilles Tendon as a Transverse Isotropic Plate}

The tendon model considered in this study presents a random distribution of fibers oriented in the same direction $(x$ in Fig. 2). This implies the existence of symmetry along the fiber orientation, which corresponds to a hexagonal system or as mentioned above transverse isotropy. The Christoffel's tensor $\mathrm{C}_{\mathrm{ij}}$ describing such system is composed of 5 independent elastic constants: $\mathrm{C}_{11}, \mathrm{C}_{33}, \mathrm{C}_{13}, \mathrm{C}_{55}$ and $\mathrm{C}_{66}$. In an infinite transverse isotropic medium the elastic constants $\mathrm{C}_{11}$ and $\mathrm{C}_{33}$ can be determined from the measurement of longitudinal ultrasound phase velocities along the perpendicular and parallel direction respectively [2]. Moreover, the elastic constants $\mathrm{C}_{55}$ and $\mathrm{C}_{66}$ can be deduced from the phase velocity measurement of shear waves propagating either parallel or perpendicular to the fiber direction with a polarization perpendicular to the fibers [8]. However, in a transverse isotropic plate the relation between phase velocity and elasticity is more complex.

The problem of wave propagation along a transverse isotropic plate of thickness $h$ surrounded by liquid has already been studied by Dayal et al. [10]. In this work it was demonstrated that guided wave propagation along the fiber direction can be described by a superposition of anti-symmetric and symmetric modes similar to what happens in an isotropic plate where Lamb modes are present. In the framework of SSI, due to the frequencies involved and the source symmetry (i.e. the radiation force mainly induces displacements perpendicular to the plate), energy will propagate as a zero order antisymmetric mode [11], [12]. Therefore attention will be focused 
on the secular equation (1) corresponding to the anti-symmetric modes [10]:

$$
\begin{aligned}
& \frac{G_{p} H_{m}}{G_{m} H_{p}}-\frac{\tan \left(k_{p} h / 2\right)}{\tan \left(k_{m} h / 2\right)}+ \\
& \quad+i \frac{\rho_{l i q} \omega^{2}}{k_{\text {liq }}} \frac{\cot \left(k_{p} h / 2\right)}{G_{m}}\left(\frac{H_{m}}{H_{p}}-1\right)=0
\end{aligned}
$$

Where $H_{p, m}, G_{p, m}$ and $k_{p, m}$ are given by:

$$
\begin{gathered}
H_{p, m}=k+k_{p, m}\left(\frac{\rho \omega^{2}-C_{11} k_{p, m}^{2}-k^{2} C_{55}}{\left(C_{55}+C_{13}\right) k_{p, m} k}\right) \\
G_{p, m}=C_{11} k_{p, m}+C_{13} k\left(\frac{\rho \omega^{2}-C_{11} k_{p, m}^{2}-k^{2} C_{55}}{\left(C_{55}+C_{13}\right) k_{p, m} k}\right) \\
k_{p, m}^{2}=\frac{k^{2}}{2}\left(-B \pm \sqrt{B^{2}-4 D}\right) \\
k_{l i q}^{2}=k^{2}\left(\frac{\omega^{2}}{k^{2} c_{l i q}^{2}}-1\right)
\end{gathered}
$$

In (2) to (4) $k$ is the Lamb wave number defined as the ratio between phase velocity and the angular frequency $(\omega=2 \pi f), \rho$ is the plate's density, $\rho_{\text {liq }}$ is the liquid's density, $c_{\text {liq }}$ is the liquid's sound speed and the subscripts $p$ and $m$ correspond to the plus and minus sign in (4) respectively. B and D terms are presented in (6) and (7) respectively.

$$
\begin{gathered}
B=\frac{-\left(C_{55}+C_{13}\right)^{2}-\left(\rho \omega^{2} / k^{2}\right)\left(C_{55}+C_{11}\right)}{C_{11} C_{55}}+ \\
+\frac{C_{11} C_{33}+C_{55}^{2}}{C_{11} C_{55}} \\
D=\frac{\rho \omega^{2}}{k^{2}} \frac{\rho \omega^{2} / k^{2}-C_{33}}{C_{11} C_{55}}-\frac{\rho \omega^{2}}{k^{2}} \frac{1}{C_{11}}+\frac{C_{33}}{C_{11}}
\end{gathered}
$$

Thus, by solving (1) using a Nelder-Mead minimization method the theoretical dispersion curve for the anti-symmetric modes propagating parallel to the fibers of a transverse isotropic plate can be computed [11], [12]. Is important to note that (1) only depends on the plate thickness $h$ and the elasticity constants $\mathrm{C}_{11}, \mathrm{C}_{33}, \mathrm{C}_{13}$ and $\mathrm{C}_{55}$ because $\rho$ and $\rho_{\text {liq }}$ were assumed to be equal to $1100 \mathrm{~kg} / \mathrm{m}^{3}$ while $c_{\text {liq }}$ was assumed to be $1500 \mathrm{~m} / \mathrm{s}$. Thus, by fitting the theoretical model given in (1) in a least square sense to the experimental dispersion curves the elasticity constants were retrieved.

In Fig. 3 the least mean square fit is presented in black full line along with the experimental dispersion curves. During the fitting process the plate thickness $h$ and the elasticity constants $\mathrm{C}_{11}$ and $\mathrm{C}_{33}$ were held fixed while the elasticity constant $\mathrm{C}_{13}$ was allowed to vary under the following conditions: $\sqrt{ }\left(C_{11} C_{33}\right)$ $\geq \mathrm{C}_{13} \geq \min \left(\mathrm{C}_{11}, \mathrm{C}_{33}\right)$. The values for $\mathrm{C}_{11}$ and $\mathrm{C}_{33}$ were assumed to be equal to $2.89 \mathrm{GPa}$ and $3.51 \mathrm{Gpa}$ respectively, values reported in the literature for the Achilles tendon [1], [2], while the values for $h$ were measured directly from the B-Mode image of each tendon. A very good agreement between the model and the experimental data is observed. This fact is supported by a mean correlation coefficient of $0.998 \pm 0.001$. Mean elasticity constants of $\mathrm{C}_{13}=3.1 \pm 0.3 \mathrm{GPa}$ and $\mathrm{C}_{55}=$ $1029 \pm 413 \mathrm{kPa}$ were obtained by averaging the values retrieved for the four tendons. These values are in good agreement with the values reported in the literature [1]-[3].

Along the perpendicular direction the secular equation (8) for the anti-symmetric modes is the following:

$$
\begin{aligned}
\frac{4 k_{L} k_{T} k^{2}}{\left(k_{T}^{2}-k^{2}\right)^{2}}+ & \frac{\tan \left(k_{L} h / 2\right)}{\tan \left(k_{T} h / 2\right)}+ \\
& +i \frac{\rho \cdot k_{L} \cdot \rho_{\text {liq }} \omega^{4}}{k_{\text {liq }}\left(k_{T}^{2}-k^{2}\right)^{2}} \frac{\cot \left(k_{T} h / 2\right)}{C_{66}^{2}}=0
\end{aligned}
$$

Where $k_{L}$ and $k_{T}$ are given by:

$$
\begin{aligned}
& k_{T}^{2}=k^{2}\left(\frac{\rho \omega^{2}}{k^{2}} \frac{1}{C_{66}}-1\right) \\
& k_{L}^{2}=k^{2}\left(\frac{\rho \omega^{2}}{k^{2}} \frac{1}{C_{11}}-1\right)
\end{aligned}
$$

Thus, contrary to what happens along the parallel direction, for the perpendicular direction, waves obey the leaky Lamb wave equation for an isotropic plate of elasticity constants $C_{11}$ and $\mathrm{C}_{66}$. By assuming $\rho, h$ and $\mathrm{C}_{11}$ to be constant the only parameter left to vary is $\mathrm{C}_{66}$. The theoretical dispersion curves are presented in Fig. 4 in green dashed line. In Fig. 4 it can be observed that the leaky Lamb wave dispersion model does not fully explain the experimental dispersion curve.

As mentioned above, viscosity may play a role in wave dispersion. Thus, in the perpendicular direction, a viscoelastic plate was considered. To introduce viscosity the elastic constant $\mathrm{C}_{66}$ was no longer considered as a real number but a complex number instead. Its frequency dependence was assumed to obey a Voigt model: $\mathrm{C}_{66}=\mu_{1}+\mathrm{i} \omega \mu_{2}$ with $\mu_{l}$ elastic component and $\mu_{2}$ viscous component. In Fig. 4 the theoretical dispersion curves are presented in full black line along with the experimental dispersion curves. A very good agreement between the model and the experimental data was observed for 
the frequencies above $500 \mathrm{~Hz}$. Mean elasticity and viscosity values of $\mu_{1}=19 \pm 2 \mathrm{kPa}$ and $\mu_{2}=2.5 \pm 0.6 \mathrm{~Pa}$.s were obtained by averaging the values retrieved for the four tendons. The elasticity values are in good agreement with the literature [3], while the viscosity values are consistent with the ones reported for other tissue such as muscle [8].
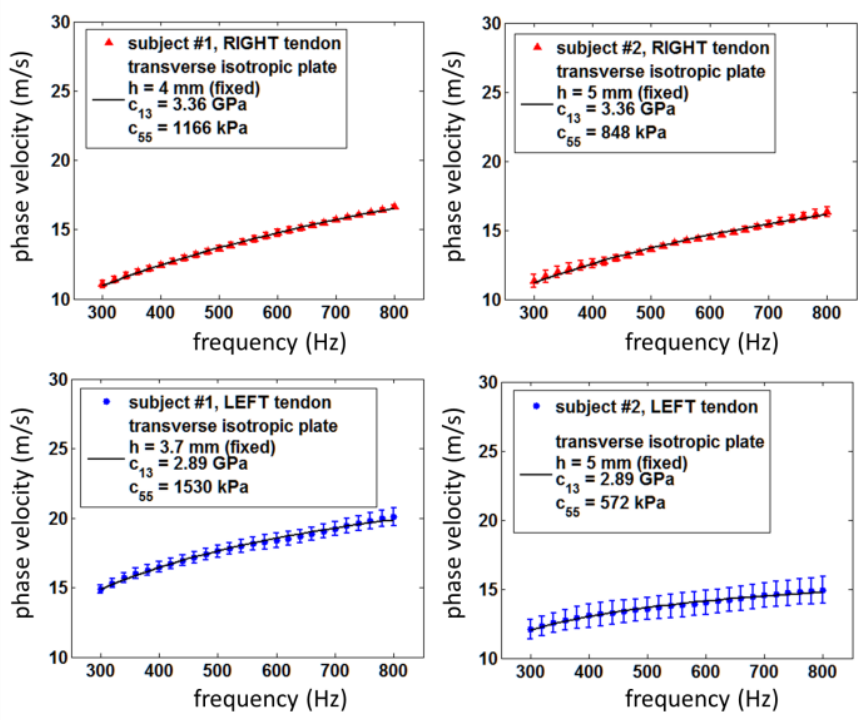

Fig. 3. Shear wave dispersion curves for the propagation direction parallel to the fibers. In black full line the least mean square fit of the transverse isotropic plate model.
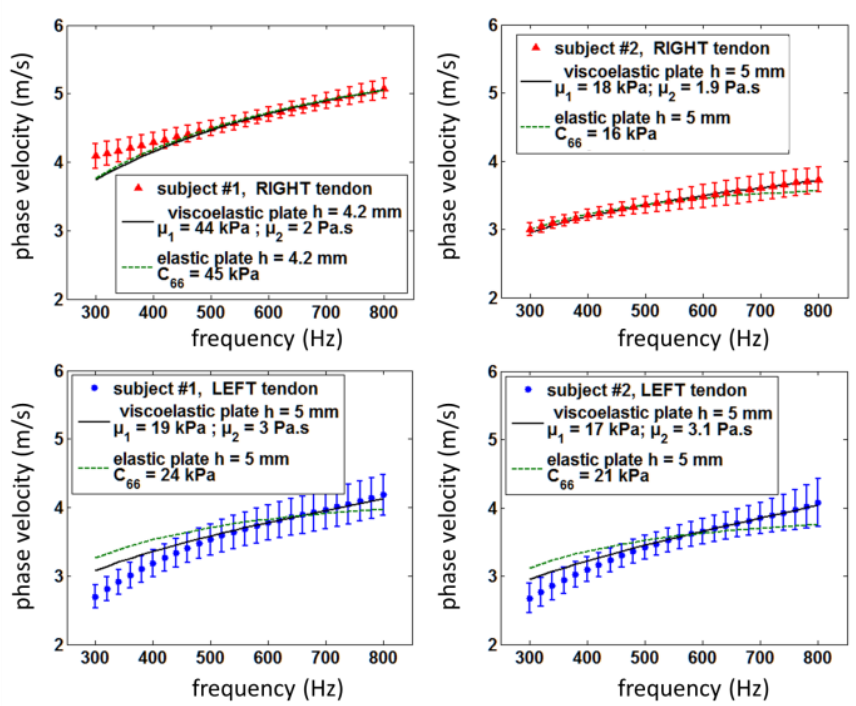

Fig. 4. Shear wave dispersion curves for the propagation direction perpendicular to the fibers. In black full line and green dashed line the fit corresponding to the viscoelastic and elastic transverse isotropic plate are presented respectively.

\section{DISCUSSION \& CONCLUSIONS}

In this study wave dispersion was measured in vivo in four Achilles tendons by using the SSI technique and the concept of
SWS. The results showed the importance of a dispersion model in order to properly estimate the tendon elasticity. To model the observed dispersive behavior the tendon was assumed to act as a transverse isotropic plate, guiding waves along both propagation directions. Parallel to the fiber direction, the elastic plate model completely explains the experimental dispersion curve, however this was not the case for the perpendicular direction were the tendon viscosity had to be taken into account. This fact can be explained by either viscosity is negligible along the parallel direction (i.e. viscosity is also transverse isotropic) or the dispersion curve is simply not affected by viscosity as it was already been demonstrated in [12]. Further studies are being carried out to clarify this point.

\section{ACKNOWLEDGMENT}

This work was supported by LABEX WIFI (Laboratory of Excellence within the French Program "Investments for the Future") under references ANR-10-LABX-24 and ANR-10IDEX-0001-02 PSL and the Agence Nationale pour la Recherche (ANR).

\section{REFERENCES}

[1] B.K. Hoffmeister, S.M. Handley, S.A. Wickline, and J.G. Miller, "Ultrasonic determination of the anisotropy of Young's modulus of fixed tendon and fixed myocardium", J. Acoust. Soc. Am. 100(6), 39333940 (1996).

[2] P.L. Kuo, P.C. Li, and M.L. Li, "Elastic properties of tendon measured by two different approaches", Ultrasound Med. Biol. 27(9), 1275-1284 (2001).

[3] S. Aubry et al., "Biomechanical properties of the calcaneal tendon in vivo assessed by transient shear wave elastography", Skeletal Radiol. DOI 10.1007/s00256-013-1649-9 (2013).

[4] M. Tanter, D. Touboul, J.-L. Gennisson, J. Bercoff, and M. Fink, "Highresolution quantitative imaging of cornea elasticity using supersonic shear imaging", IEEE Trans. Med. Imaging 28(12), 1881-1893 (2009).

[5] M. Couade et al., "Quantitative assessment of arterial wall biomechanical properties using shear wave imaging", Ultrasound Med. Biol. 36(10), 1662-1676 (2010).

[6] T. Deffieux, G. Montaldo, M. Tanter, and M.Fink, "Shear Wave Spectroscopy for In Vivo Quantification of Human Soft Tissues Viscoelasticity", IEEE Trans. Med. Im. 28(3), 313-322 (2009).

[7] J. Bercoff, M. Tanter, and M. Fink, "Supersonic shear imaging: A new technique for soft tissue elasticity mapping", IEEE Trans. Ultrason. Ferroelectr. Freq. Control 51(4), 396-409 (2004).

[8] J.-L. Gennisson et al., "Viscoelastic and anisotropic mechanical properties of in vivo muscle tissue assessed by supersonic shear imaging", Ultrasound Med. Biol. 36(5), 789-801 (2010).

[9] I.Z. Nenadic et al., "On Lamb and Rayleigh wave convergence in viscoelastic tissues", Phys. Med. Biol. 56, 6723-6738 (2011).

[10] V. Dayal, V.K. Kinra, "Leaky lamb waves in anisotropic plate I: an exact solution and experiments", J. Acoust. Soc. Am. 85(6), 2268-2276 (1989).

[11] J. Brum et al., "Application of 1D transient elastography for the shear modulus assessment of thin layered soft tissue: comparison with Supersonic Shear Imaging technique", IEEE Trans. Ultras. Ferroelec. Freq. Control 59(4), 703-714 (2012).

[12] T.-M. Nguyen, M. Couade, J. Bercoff, and M. Tanter, "Assessment of viscous and elastic properties of sub-wavelength layered soft tissues using shear wave spectroscopy: Theoretical framework and experimental in vitro experimental validation," IEEE Trans. Ultrason. Ferroelectr. Freq. Control 58(11), 2305-2315 (2011). 